\title{
Percepção da assistência prestada pela equipe multiprofissional da sala vermelha de um hospital de urgência e emergência do interior de Rondônia
}

\author{
Perception of assistance provided by the multiprofessional team in the red room of the \\ hospital of emergency and emergency in the Rodonia inland
}

Percepción de asistencia proporcionada por el equipo multiprofesional en la sala roja del hospital de emergencia y emergencia en el interior de Rodonia

Angélica Inácio da Cruz Oliveira ${ }^{1 *}$, Angela Antunes de Morais Lima ${ }^{1}$, Emanoela Maria Rodrigues de Sousa1, Poliana Deyse Pereira Gouvêa ${ }^{1}$, Cassia de Oliveira Pinto Rosa ${ }^{1}$, Tatiane Maestá1.

\section{RESUMO}

Objetivo: Analisar a assistência prestada no setor de urgência e emergência (sala de estabilização), através do perfil da equipe multiprofissional. Métodos: Pesquisa de características qualitativas, realizada em agosto de 2019 em uma instituição hospitalar de urgência e emergência. Para análise dos dados foi utilizado o programa Microsoft Office Excel® versão 2010. Resultados: Os profissionais participantes da pesquisa foram compostos em sua maioria $56 \%$ pelo gênero masculino. $76 \%$ dos entrevistados possuem nível superior, destes apenas $12 \%$ possuem pós-graduação ou especialização em urgência e emergência. $54 \%$ dos participantes não realizam cursos de aprimoramento na área de urgência e emergência, e $52 \%$ nunca realizaram cursos como Suporte Avançado de Vida no Trauma (ATLS), Suporte Avançado de Vida Cardiovascular (ACLS) e atendimento pré-hospitalar (APH). 68\% responderam que a unidade onde trabalham não oferece cursos de atualização em urgência e emergência e $84 \%$ não participam de tais programas. $84 \%$ da equipe entrevistada se sentem aptos a atuar no setor de urgência e emergência mesmo sem qualificação adequada. Conclusão: Diante dos resultados encontrados na atual pesquisa se faz necessário o complexo hospitalar incentivar e investir em capacitações e treinamentos dos profissionais atuantes no pronto socorro visando qualificar a equipe para melhorar o serviço prestado.

Palavras-Chave: Credenciamento, Equipe de assistência ao paciente, Emergências.

\begin{abstract}
Objective: Objective: To analyze the assistance provided in the urgency and emergency sector (stabilization room), through the profile of the multiprofissional team. Methods: Qualitative research carried out in August 2019 in an emergency hospital institution. For data analysis we used the Microsoft Office Excel® version 2010 program. Results: The professionals participating in the research were mostly $56 \%$ male. $76 \%$ of respondents have a college degree, of these only $12 \%$ have postgraduate or specialization in urgency and emergency. $54 \%$ of respondents did not take emergency and improvement courses and $52 \%$ had never taken courses such as Advanced Trauma Life Support (ATLS), Advanced Cardiovascular Life Support (ACLS) and Prehospital Care (APH). 68\% responded that the unit where they work does not offer urgent and emergency refresher courses and $84 \%$ do not participate in such programs. $84 \%$ of the interviewed staff feel able to work in the urgency and emergency sector even without proper qualification. Conclusion: Facing the published outcomes in this research its necessary investments in training and professional qualifications for the professionals that work in emergency room to qualify and improve the assistance.
\end{abstract}

Keywords: Accreditation, Patient care team, Emergencies.

\section{RESUMEN}

Objetivo: Analizar la asistencia brindada en el sector de urgencias y emergencias (sala de estabilización), a través del perfil del equipo multiprofesional. Métodos: Investigación cualitativa realizada en agosto de 2019 en un hospital de urgencias. Para el análisis de datos, se utilizó el programa Microsoft Office Excel® versión

${ }^{1}$ Complexo Hospitalar Regional de Cacoal (COHREC), Cacoal-RO.

*E-mail: angelicaoliveira2806@gmail.com 
2010. Resultados: los profesionales que participaron en la investigación eran en su mayoría $56 \%$ hombres. El $76 \%$ de los encuestados tiene un título universitario, de estos, solo el 12\% tiene posgrado o especialización en urgencias y emergencias. El $54 \%$ de los participantes no toma cursos de mejora urgente y de emergencia, y el $52 \%$ nunca ha tomado cursos como Advanced Trauma Life Support (ATLS), Advanced Cardiovascular Life Support (ACLS) y Prehospital Care (APH). El 68\% respondió que la unidad donde trabajan no ofrece cursos de actualización urgente y de emergencia y el $84 \%$ no participa en dichos programas. El $84 \%$ del personal entrevistado se siente capaz de trabajar en el sector de urgencias y emergencias, incluso sin la calificación adecuada. Conclusión: Dados los resultados encontrados en la investigación actual, es necesario que el complejo hospitalario aliente e invierta en calificaciones y capacitación de profesionales que trabajan en la saleta de emergencias para calificar al equipo para mejorar el servicio brindado.

Palabras clave: Acreditación, Equipo de atención al paciente, Emergencias.

\section{INTRODUÇÃO}

O setor de urgência e emergência tem como propósito um método de abordagem qualificada dos usuários, objetivando organização do setor, resolução dos problemas relacionados à saúde, mudanças das práticas de saúde dos trabalhadores, entre outros, favorecendo uma relação de confiança e compromisso entre usuários e a equipe de saúde (BRASIL, 2008).

O pronto socorro deve ser um serviço multidisciplinar de especialistas em tratar pacientes em estado grave, lembrando que é uma das áreas mais críticas e congestionadas dentro do hospital, onde situações inesperadas ocorrem (VIANA PP e SANTOS FPL, 2009).

Para Silva CR e Ferreira MA (2011), o negligenciamento/subutilização do uso da tecnologia no cuidado, assim como o uso não fundamentado da mesma pode afetar de forma negativa a saúde do cliente de modo irreversível, visto que o paciente se encontra num quadro em que suas funções vitais estão suscetíveis a rápidas mudanças, condições que se agravam com a assistência prestada por profissionais não qualificados.

O trabalho prestado nos serviços de urgência e emergência hospitalar se faz necessário um vasto conhecimento de saúde e domínio dos profissionais sobre o processo de trabalho, nestas unidades de atendimento se exige pensar rápido com agilidade, tendo competência e capacidade para que os problemas tenham maior resolubilidade, pois, neste ambiente o tempo é limitado, há sobrecarga de atividades o que exige, muitas vezes, que o profissional atue com rapidez para afastar o risco de morte iminente dos pacientes com estado de saúde critico (ALMEIDA PJS e PIRES DEP, 2007).

Sendo assim, se faz necessário qualificar a assistência e promover a capacitação continuada das equipes de saúde atuantes no setor de Atenção às Urgências, em acordo com os princípios da integralidade e humanização (BRASIL, 2003), possibilitando que o setor tenha profissionais especializados, sendo capacitados para $O$ atendimento das urgências e emergências, e promover treinamento e educação permanente de acordo com as atividades desenvolvidas (BRASIL, 2014).

Quando os profissionais prestam atendimento em situações de urgência, e não conseguem visualizar a trajetória dos usuários e as dificuldades pelas quais passam para que chegasse até a satisfação de suas necessidades de saúde, sendo que é de extrema importância essa compreensão, para tornar o atendimento mais acolhedor, utilizando uma abordagem que leve à solução competente e satisfaça o usuário (GARLET, ER, et al.,2009).

Nesse sentido, a literatura aponta que os profissionais de saúde atuantes nos serviços de urgência e emergência necessitam desenvolver habilidades que garantam competência técnico-científica e postura acolhedora e humanizada com os usuários (SILVA, PL, et al.,2016).

O Serviço de Urgência e Emergência deve contar com profissionais especializados, sendo capacitados para o atendimento das urgências e emergências, e promover treinamento e educação permanente de acordo com as atividades desenvolvidas (BRASIL, 2014).

Esta pesquisa buscou analisar a assistência prestada na sala vermelha do Hospital de Urgência e Emergência identificando o perfil da equipe multiprofissional atuante na sala, sua relevância se da devido a poucos estudos realizados envolvendo a equipe multiprofissional. 
Serviço que, numa linha muito tênue, impõe ao profissional a necessidade permanente de atualizações, investimentos em cursos e treinamentos, com vistas às implementações cabíveis no avanço da prestação do serviço de urgência e emergência.

Quiçá dizer, ainda, que o bom desempenho profissional acarretará com a eficiência da performance em redução de gastos e implementação da qualidade de vida do usuário.

\section{MÉTODOS}

Trata-se de um estudo descritivo de característica qualitativa, realizado em uma instituição hospitalar localizada no cone sul do Estado de Rondônia, com a participação da equipe multiprofissional atuante no setor da sala vermelha, conforme a escala elaborada pela coordenação de enfermagem, médica e de fisioterapia.

A coleta de dados foi realizada pelas próprias pesquisadoras, na primeira semana de agosto de 2019, os sujeitos participantes foram 25 profissionais da equipe multiprofissional da Sala Vermelha, foram incluídos na pesquisa todos os profissionais da equipe multiprofissional que atuam há mais de três meses na sala vermelha de um hospital de Urgência e Emergência de um município de Rondônia, aqueles que aceitarem participar da pesquisa através da assinatura do termo de consentimento livre e esclarecido (TCLE) voluntariamente.

Foram excluídos os Profissionais que estiverem afastados ou sob licença durante o período de pesquisa, Profissionais que se negarem em responder o questionário em seu decorrer, aqueles que não assinarem o TCLE.

Para análise dos dados foi utilizado o programa Microsoft Office Excel® versão 2010. Esta pesquisa aconteceu posteriormente à aprovação pelo Comitê de Ética em Pesquisa da (CEP) da Faculdade de Ciências Biomédicas de Cacoal (FACIMED), sob o parecer no CAAE 97260718.5.0000.5298. Respeitaram-se todas as condições impostas pela Resolução CNS no-466/2012 do Conselho Nacional de Saúde (CNS) (BRASIL, 2012).

\section{RESULTADO E DISCUSSÃO}

De acordo com o estudo realizado a amostra foi composta em sua maioria pelo gênero masculino 14 (56\%) e 11 (44\%) pelo gênero feminino, à faixa etária predominante de idade foi de 27 a 53 anos sendo que 19 (76\%) tinham entre 27 e 39 anos, e apenas 6 (24\%) com idade igual ou superior que 40 anos, foi avaliada a equipe multiprofissional atuantes na sala vermelha composta por médicos $7(28 \%)$, enfermeiros $5(20 \%)$, fisioterapeutas $1(1 \%)$ e técnico de enfermagem $12(48 \%)$, onde $6(24 \%)$ possuíam nível médio de escolaridade e 19 (76\%) nível superior, dentre estes 13 (52\%) tinham pós-graduação, porém, apenas 3 (12\%) na área de urgência e emergência.

Ao analisar o tempo de atuação em Pronto Socorro (PS) percebeu-se que $15(60 \%)$ tem entre 3 meses e 5 anos de PS e 10 (40\%) entre 6 anos e 18 anos. Referente ao tempo de formação acadêmica na área de atuação dos profissionais teve variação de 5 meses a 30 anos de formação, sendo que 13 (52\%) com tempo de formação de 5 meses a 6 anos e $12(48 \%)$ de 7 anos a 30 anos de formação. Conforme mostra a Tabela 1. 
Tabela 1 - Distribuição de acordo com as variáveis do Perfil da equipe multiprofissional atuantes na sala vermelha do hospital de urgência e emergência do interior de Rondônia.

\begin{tabular}{|c|c|c|}
\hline Variáveis & $\mathbf{N}^{\circ}$ & $\%$ \\
\hline \multicolumn{3}{|l|}{ Sexo } \\
\hline Feminino & 11 & 44 \\
\hline Masculino & 14 & 56 \\
\hline \multicolumn{3}{|l|}{ Idade } \\
\hline De 27 a 39 anos & 19 & 76 \\
\hline$<\mathrm{ou}=\mathrm{a} 40$ anos & 6 & 24 \\
\hline \multicolumn{3}{|l|}{ Categoria profissional } \\
\hline Medico & 7 & 28 \\
\hline Enfermeiro & 5 & 20 \\
\hline Fisioterapeuta & 1 & 4 \\
\hline Tec. enfermagem & 12 & 48 \\
\hline \multicolumn{3}{|l|}{ Nível de escolaridade } \\
\hline Médio & 6 & 24 \\
\hline Superior & 19 & 76 \\
\hline \multicolumn{3}{|l|}{ Pós-graduação } \\
\hline Sim & 13 & 52 \\
\hline Não & 12 & 48 \\
\hline \multicolumn{3}{|c|}{$\begin{array}{l}\text { Especialização/ pós-graduação em } \\
\text { urgência e emergência }\end{array}$} \\
\hline Sim & 3 & 12 \\
\hline Não & 22 & 88 \\
\hline \multicolumn{3}{|c|}{ Tempo de formação acadêmica } \\
\hline De 5 meses a 6 anos & 13 & 52 \\
\hline De 7 anos a 30 anos & 12 & 48 \\
\hline \multicolumn{3}{|c|}{$\begin{array}{l}\text { Há quanto tempo atua no pronto } \\
\text { socorro? }\end{array}$} \\
\hline De3 meses a 5 anos & 15 & 60 \\
\hline De 6 anos a 18 anos & 10 & 40 \\
\hline Total & 25 & $100 \%$ \\
\hline
\end{tabular}

Fonte: Oliveira AIC, et al., 2020.

Nesta pesquisa houve predominância do gênero masculino, índices parecidos foram encontrados por Costa FSO et al, (2019); onde observa - se que a população estudada, teve predominância do gênero masculino, o que correspondeu a $65 \%$ de sua amostra total, resultados estes que divergem do estudo de Lopes FS e Andreoti MT (2014), cujo evidenciaram que a maioria dos profissionais $95,1 \%$ eram do sexo feminino e apenas $4,9 \%$ era do sexo masculino e com idade entre 31 e 40 anos, sendo que $52,1 \%$, formada em média há 9 anos.

Também foi evidenciado a predominância do sexo feminino atuante no setor de urgência e emergência no estudo de Jorge VC e Barreto MS (2012) e Custodio IL, et al. (2011), onde 92,3\% e 79,2\% eram mulheres respectivamente, o mesmo autor também ressaltou que a idade dos participantes variou de 23 a 65 anos, 
com média de idade 45 anos, onde a predominância da faixa etária de 40 a 50 anos foi de $36,1 \%$, reforçada por Jorge VC e Barreto MS (2012), com resultado onde 52,6\% eram profissionais com mais de 40 anos.

Com os resultados expostos acima vemos um cenário na urgência e emergência diferente do encontrado em outros setores e em outros estudos, onde o sexo masculino passa a se sobrepor ao feminino, talvez por ser um setor onde se exija um maior controle emocional e maior esforço físico.

Analisando formação escolar, Silva KR e Pires RCCPA (2011), constataram que $63 \%$ dos entrevistados em sua pesquisa possuem ensino médio completo e $17 \%$ dos de nível superior possuem pós-graduação, o que diverge dos resultados da atual pesquisa onde a prevalência do nível de escolaridade foi de superior completo.

Já Cavalcante AKCB, et al. (2014), evidenciou que 36,6\% dos entrevistados fizeram curso superior que se assemelha aos dados encontrados no estudo de Jorge VC e Barreto MS (2012), cujo identificou que 77,27\% fizeram curso de graduação e, deste total apenas 5,88\% fizeram pós-graduação.

Costa FSO, et al. (2019), observou em seu estudo que a titulação dos profissionais participantes teve prevalência a de especialista, com $38,75 \%$ dos participantes, enquanto que, 33,75\% apresentam apenas a formação de nível médio e corresponde a categoria profissional de técnico de enfermagem.

A atual pesquisa evidencia que os profissionais que compõe a equipe multiprofissional do setor de urgência e emergência estão buscando uma melhor formação acadêmica, porém, sem especializações na área que atuam no momento, o que não irá influenciar de forma positiva e muito menos aperfeiçoar a assistência que será prestada neste setor que é a porta de entrada hospitalar, na qual se recebe o paciente critico necessitando de uma intervenção rápida e de qualidade.

Almeida PJS e Pires DEP (2006) revelaram em seu estudo que a maioria dos profissionais atua na emergência por ser uma oportunidade de emprego e não por escolha pessoal, e que a equipe composta por esses profissionais era muito jovem mostrando que existe uma curta permanência no serviço, pois eles vivenciando diariamente sentimentos de frustação e impotência, gerando enormes cargas físicas e psíquicas, levando-os a se afastarem do setor de urgência e emergência com um menor período de tempo.

No estudo de Cavalcante AKCB, et al. (2014), 34,1\% dos participantes trabalhavam de 2 a 5 anos, e Jorge VC e Barreto MS (2012), onde 55,3\% apresentavam tempo superior a 10 anos de pronto socorro diferente dos dados obtidos nesta pesquisa onde a maioria dos profissionais atuam na unidade de urgência e emergência por um período menor que 6 anos.

Dados mais próximos da referida pesquisa foram encontrados por Silva KR e Pires RCCPA (2011), em seu estudo onde $21 \%$ a minoria dos profissionais tinha de 7 a 10 anos de atuação PS, no estudo de Silva AP, et al. (2012), um percentual menor (43,2\%) atuava pelo tempo de 1 a 5 anos no setor de urgência e no estudo de Silva KR e Pires RCCPA (2011), 38\% dos participantes, afirmaram possuir de 4 a 6 anos de formados, resultados inferiores aos encontrados na atual pesquisa.

Contudo percebemos ao decorrer da aplicação do questionário que a maioria dos profissionais ali atuantes foram inseridos sem treinamento prévio, e mesmo com anos de experiência demonstram insegurança e dificuldades diante de situações criticas.

Na presente pesquisa realizada no setor de pronto socorro percebemos que 14 (56\%) dos participantes não realizam cursos de aprimoramento na área de urgência e emergência, sendo realizado por apenas 11 (44\%) dos profissionais, sendo que 13 (52\%) nunca realizaram cursos como Suporte Avançado de Vida no Trauma (ATLS), Suporte Avançado de Vida Cardiovascular (ACLS), e atendimento pré-hospitalar (APH).

Dos entrevistados $17(68 \%)$ responderam que a unidade onde trabalham não oferece cursos para atualização voltados para urgência e emergência apenas 8 (32\%) disseram que lhes são ofertados tais cursos, entretanto 21 (84\%) não participam de programas de educação continuada.

Mesmo atuando no setor de urgência e emergência 14 (56\%) da equipe multiprofissional não conhecem os protocolos do setor. 21 (84\%) refere ser aptos a atuar em um OS, e 21 (84\%) responderam que seu conhecimento pratico e teórico lhes oferece confiança para exercer sua funções e tal setor, assim como 23 (92\%) se sentem capaz de tomar decisões rápidas e coerentes diante de uma situação de risco eminente de morte de um paciente. Conforme mostra a Tabela 2. 
Tabela 2 - Distribuição dos resultados quanto ao conhecimento e credenciamento da equipe multiprofissional atuantes na sala vermelha do hospital de urgência e emergência do interior de Rondônia.

\begin{tabular}{ccc}
\hline Variáveis & $\mathbf{N}^{\circ}$ & $\%$ \\
\hline $\begin{array}{c}\text { Realiza cursos de aprimoramento em urgência e } \\
\text { emergência? }\end{array}$ & & \\
\hline Sim & 11 & 46 \\
Não & 14 & \\
\hline Realizou cursos de APH; ATLS e ACLS? & 12 & 48 \\
\hline Sim & 13 & 52 \\
\hline Não & & \\
\hline A unidade onde atua oferece cursos e atualizações \\
em urgência e emergência?
\end{tabular}

Participa de programas de educação continuada?

\begin{tabular}{ccc}
\hline Sim & 4 & 16 \\
Não & 21 & 84 \\
\hline $\begin{array}{c}\text { Conhece os protocolos do setor onde atua? } \\
\text { Sim }\end{array}$ & 11 & 44 \\
\hline Não & 14 & 56 \\
\hline Se sente apto a atuar no pronto socorro? & & 84 \\
\hline Sim & 21 & 16 \\
\hline Não & 4 & \\
\hline
\end{tabular}

Seu conhecimento teórico e prático lhe oferece segurança para atuar no pronto socorro?

$\begin{array}{lcc}\text { Sim } & 21 & 84 \\ \text { Não } & 4 & 16\end{array}$

Se sente capaz de tomar decisões rápidas e coerentes em situações de risco eminente de morte do paciente?

\begin{tabular}{cccc}
\hline Sim & 23 & 92 \\
Não & 2 & 8 \\
\hline Total & 25 & 100
\end{tabular}

Legenda: APH: atendimento pré-hospitalar. ATLS: Suporte Avançado de Vida no Trauma. ACLS: Suporte Avançado de Vida Cardiovascular. Fonte: OLIVEIRA AIC, et.al. 2020. 
Para que a assistência adequada seja prestada ao cliente em estado grave é necessária uma equipe capacitada tanto tecnicamente quanto emocionalmente, para ter competência ética e conduzir situações com risco eminente de morte da melhor maneira possível, é preciso que estes profissionais estejam sempre buscando uma educação continuada, pois para atuar no PS necessitam estar capacitado para exercer suas funções, o que não é a realidade evidenciada pela atual pesquisa, pois poucos profissionais buscam por atualizações, aperfeiçoamento ou cursos na área de urgência e emergência.

O Conselho Federal de Enfermagem (COFEN, 2007) ressalta que é direito dos profissionais buscarem o aprimoramento de seus conhecimentos técnicos, científicos e culturais que dão sustentação a prática profissional (RESOLUÇÃO 311/2007, cap.1, art. 2), porém, em sua maioria os profissionais estão buscando formação em outras áreas que não são voltadas para o setor de urgência e emergência.

O estudo de Woiski ROS e Rocha DLB (2010); diz-nos que o trabalho deve ser de forma harmoniosa, rápida e em total sincronia, objetivando extinguir sequelas ou agravos. Porém, os colaboradores atuantes no pronto socorro exercem suas funções de acordo com a rotina que aprenderam com os profissionais que atuam há mais tempo no serviço julgam ser a correta, sendo assim não sabem se tais rotinas realmente estão adequadas como preconiza o protocolo implantado no setor.

Os resultados encontrados na pesquisa de Costa FSO et al, (2019); apontam que a maioria dos profissionais de saúde reconhecem a relevância da utilização dos protocolos estabelecidos pela OMS, como métodos que propiciam práticas assistenciais mais seguras, estando representado no percentual de $92,31 \%$ de tendência positiva das respostas obtidas, referente a questão.

O que diverge dos dados adquiridos neste estudo onde a maioria dos profissionais que compõem a equipe da sala vermelha não conhecem os protocolos implantados, e em nenhum momento buscou conhecer os protocolos, já que os mesmos não ficam disponíveis no setor como deveras seria, mostrando a falta de interesse dos mesmos para com o conhecimento das normas e rotinas que desempenham de forma errônea diariamente.

A Política Nacional de Atenção às Urgências traz que é necessário qualificar a assistência e promover a capacitação continuada das equipes de saúde do Sistema Único de Saúde (SUS) e na Atenção às Urgências, respeitando os princípios da integralidade e humanização ofertando capacitação e educação continuada para as equipes de todos os níveis de atenção (BRASIL, 2006).

Diferentemente do que a Política Nacional de Atenção às Urgências nos trás a equipe entrevistada na pesquisa mostra em sua supremacia o hospital não oferece cursos de atualizações ou treinamentos, não contribuindo assim para ampliar e aprimorar o conhecimento dos integrantes da equipe multiprofissional e para a assistência prestada pelos mesmos aos pacientes em estado de instabilidade hemodinâmica.

Percebe-se também a falta de interesse destes profissionais, pois não procuram especializações na área de urgência e emergência, não buscam participar de programas de educação continuada fora do nosocômio onde exerce sua funções. Machado MFAS, et al, (2010) diz que a educação em saúde estrategicamente procura disponibilizar conhecimentos e habilidades que auxiliem nas escolhas, induzindo uma consciência crítica.

Lopes FS e Andreoti MT (2014) ressaltam em sua pesquisa que é de grande importância a realização de treinamentos como educação continuada pra os profissionais atuantes no setor de urgência e emergência, buscando deixa-los mais confiantes no momento do atendimento, e que este setor tenha uma boa articulação com os demais níveis de atenção visando uma continuidade adequada da assistência prestada, perceberam que existe uma deficiência na organização e prestação dos serviços de saúde nos diferentes níveis de atenção, que auxilia no aumento do fluxo no setor de urgência e emergência e uma diminuição na qualidade da assistência prestada.

A excelência no atendimento nas urgências se da através de profissionais qualificados e capazes de exercer suas funções da melhor maneira possível. Durante a vivência na sala vermelha nos deparamos com diversas situações de maior complexidade, e observando e interagindo percebemos que em sua maioria os profissionais atuantes neste local não tinham conhecimento sobre o que fazer ou como agir, devido à falta de protocolos implantados e a falta de qualificação adequada da equipe multiprofissional atuante neste serviço. 
Mesmo a maioria dos profissionais entrevistados não terem especialização em urgência e emergência ou cursos voltados para este serviço, também não participam e não buscam por uma educação continuada, mesmo assim a preponderância dos entrevistados tem a percepção de que seu conhecimento teórico e prático Ihes oferece confiança para trabalhar no pronto socorro.

Entretanto ao aplicar o questionário à equipe de enfermagem em sua totalidade disse que seu conhecimento prático adquirido com o tempo de serviço prestado no setor da sala vermelha lhes oferecia confiança para atuar em meio a situações criticas, porém, seu conhecimento teórico não, e mesmo assim foram lotados no setor de urgência e emergência, mesmo que uma quantidade significante de profissionais não possuíram experiência na área de pronto socorro e alguns deixaram transparecer e afirmaram não gostar de atuar em tal setor, ainda relata serem capazes de tomar decisões rápidas e coerentes em situação de risco eminente de morte.

Diante do que foi exposto, Santos NCM (2008); considera que atendimento no PS, exige profissionais preparados e treinados com conhecimento suficiente para priorizar os atendimentos fazendo a diferença no final. O trabalho nos serviços de urgência e emergência se tornou de maior complexidade de acordo com Almeida PJS e Pires DEP (2006) devido o desenvolvimento tecnológico, da especialização e com usuários com quadros clínicos mais complexos, porém, essa complexidade não foi acompanhada, pois não houve aumento de recursos materiais, humanos e nem da estrutura destes setores.

Para Garlet ER, et al. (2009) a unidade de emergência caracteriza-se pela grande demanda por atendimentos de diversos níveis de complexidades, porém organização inadequada e falta de gestão, possibilita que esta unidade raramente desfrute de condições adequadas de trabalho, com uma quantidade adequada de profissionais e recursos materiais, possibilitando uma assistência qualificada. Estes fatores afetam os profissionais atuantes no setor e podem influenciar diretamente na assistência prestada ao cliente de forma negativa.

O Ministério da Saúde instituiu a Política Nacional de Educação Permanente em Saúde (PNEPS), buscando o fortalecimento do Sistema Único de Saúde (SUS) através da reflexão coletiva sobre o trabalho e oferta de instrumentos educativos como uma proposta de aprendizagem no trabalho, pelo trabalho e para o trabalho, a partir dos problemas enfrentados na realidade dos serviços, buscando transformar as práticas profissionais (MINISTÉRIO DA SAÚDE, 2007).

Para Ribeiro DK, et al. (2019) a PNEPS é uma política pública de fortalecimento expressivo dos trabalhadores de saúde para seu trabalho diário e apoio a qualificação da assistência prestada à sociedade. E mesmo com programas ofertados pelo ministério da saúde, os profissionais atuantes na sala vermelha que participaram da pesquisa não realizam cursos de aprimoramento e não dispõem de uma educação continuada.

\section{CONSIDERAÇÕES FINAIS}

No que tange a pesquisa realizada, evidenciou-se que a maioria dos integrantes da equipe multiprofissional atuantes em um hospital de urgência e emergência não é devidamente capacitada para exercer suas funções no setor. Faz-se necessário que o complexo hospitalar incentive e invista em capacitações e treinamentos para os profissionais já atuantes, e a inserção de novos profissionais especialistas na área de urgência e emergência, visando minimizar erros e eventos adversos com profissionais aptos para exercer suas funções. Ressalto também a importância de novas pesquisas nesta área, pois os setores de urgência e emergência são as portas de entrada do hospital e onde o paciente vai ter o desfecho do seu atendimento, se ele vive ou morrer o que dependerá da qualidade do serviço prestado.

\section{REFERÊNCIAS}

1. ALMEIDA PJS, PIRES DEP. O trabalho em emergência: entre o prazer e o sofrimento. Revista Eletrônica de Enfermagem v. 09, n. 03, p. 617 - 629. 2007.

2. BRASIL. Ministério da Saúde (BR). Política Nacional de Atenção às Urgências. 3aㅡ ed. ampl. Brasília, Ministério da Saúde; 2006. 
3. PORTARIA GM N. 1.863, DE 29 DE SETEMBRO DE 2003. Política Nacional de Atenção às Urgências. 3ª ed. ampl. Brasília, Ministério da Saúde; 2006.

4. … Rede Humaniza SUS (glossário), 2014.

5. Humaniza SUS: documento base para gestores e trabalhadores do SUS. Ministério da Saúde, Secretaria de Atenção à Saúde, Núcleo Técnico da Política Nacional de Humanização. - 4. Ed. - Brasília/DF: MS; 2008.

6. Secretaria de Atenção à Saúde. Departamento de Atenção Básica. Acolhimento à demanda espontânea: queixas mais comuns na Atenção Básica (cadernos de atenção básica), v.2, n.28. Brasília/DF: MS, 2012.p. 10-60.

7.

. Portaria GM/MS no 1.996, de 20 de agosto de 2007. Dispõe sobre as diretrizes para a implementação da Política Nacional de Educação Permanente em Saúde e dá outras providências. Diário Oficial da União 2007.

8. CAVALCANTE AKCB, et al. Perfil da equipe de enfermagem no serviço de urgência e emergência em um hospital publico. Revista Interdisciplinar, v. 7, n. 2, p. 85-94, 2014.

9. COSTA FSO, et al. Segurança do paciente: Percepção de profissionais de saúde de uma fundação pública estadual de Belém-PA. Revista Eletrônica Acervo Saúde, 11(9), e350. https://doi.org/10.25248/reas.e350.2019.

10. COFEN. Conselho Federal de Enfermagem - Resolução COFEN no 311/2007.

11. CUSTÓDIO IL, et al. Perfil sociodemográfico e clínico de uma equipe de enfermagem portadora de Hipertensão Arterial. Rev. Bras. Enferm. Brasília, v. 64, n. 1, p. 18-24, jan/fev, 2011.

12. GARLET ER, et al. Finalidade do trabalho em urgências e emergências: Concepções de profissionais. Revista LatinoAmericana de Enfermagem, 2009.

13. JORGE VC, BARRETO MS. Equipe de enfermagem e detecção de indicadores de agravamento em pacientes de pronto-socorro. Esc. Anna Nery, Rio de Janeiro, v.16, n. 4, p.767- 774. out/dez, 2012.

14. LOPES FS, ANDREOTI MT. A caracterização da demanda do setor de urgência e emergência sob a ótica dos usuários e profissionais de enfermagem. Centro Universitário Católico Salesiano Auxilium - UNISALESIANO, LinsSP. 2014

15. MACHADO MFAS, et al. Compreensão das mudanças comportamentais do usuário no Programa Saúde da Família por meio da participação habilitadora. Ciência \& Saúde Coletiva, 2010; 15(4): 2133-43.

16. RIBEIRO DK, et al. Educação Permanente em Saúde: relato de experiência do desenvolvimento de questionário avaliativo online. Revista Eletrônica Acervo Saúde, 11(18), e1644. https://doi.org/10.25248/reas.e1644.2019.

17. SANTOS NCM. Urgência e emergência para enfermagem - Do atendimento pré-hospitalar à sala de emergência. 5. Ed. São Paulo/SP: látria, 2008.p.17-25.

18. SILVA RCD, FERREIRA MA. Tecnologia na terapia intensiva e suas influências nas ações do enfermeiro. Revista da Escola de Enfermagem. USP, São Paulo, v. 45, n. 6, dez. 2011.

19. SILVA PL, et al. Acolhimento com classificação de risco do serviço de Pronto-Socorro Adulto: Satisfação do usuário. Revista da Escola de Enfermagem, USP. 2016; 50 (3): 427-432.

20. SILVA KR, PIRES RCCP. A percepção da equipe de enfermagem sobre liderança no serviço de urgência e emergência de um hospital geral de Belo Horizonte. Revista Tecer. Belo Horizonte, v. 4, n. 7, nov. 2011.

21. SILVA AP, et al. Trabalho em equipe de enfermagem em unidade de urgência e emergência na perspectiva de Kurt Lewin. Cienc. Cuid. Saude, v. 11, n. 3, p. 549-556, jul/set, 2012.

22. VIANA PP, SANTOS FPL. Assistência de Enfermagem no Pronto Socorro ao paciente em emergência cardiológica: uma revisão de literatura. Trabalho de Conclusão de Curso (Graduação em Enfermagem) - Centro Universitário do Espírito Santo - UNESC. Colatina, 2009.

23. WOISKI ROS, ROCHA DLB. Cuidado de enfermagem à criança vítima de violência sexual atendida em unidade de emergência hospitalar. Esc. Anna Nery. Rio de Janeiro/RJ, v. 14, n.1, Jan/Mar. 2010. 